\title{
SECONDARY COMPONENTS OF BINARY PULSARS \& MAGNETIC FIELD DECAY IN NEUTRON STARS
}

\author{
Shrinivas R. Kulkarni \\ Department of Astronomy, 105-24 \\ Caltech, Pasadena \\ CA 91125, U. S. A.
}

\begin{abstract}
We report the discovery of white dwarf secondaries in $0655+64$ and $0820+02$ systems. In the $2303+46$ system, we do not find any optical counterpart suggesting that the companion is another neutron star. The existence of a cool and therefore old white dwarf in the $0655+64$ system implies that the surface magnetic field of neutron stars stops decaying beyond some value(s) of field strength.
\end{abstract}

a)0655+64: Coincident with the timing position (Damashek et al. 1982) we find a $m_{R} \sim$ $22^{m}$, cool $(T \sim 8000 \mathrm{~K})$ white dwarf. The cooling age of the white dwarf $\left(\sim 2 \times 10^{9} \mathrm{y}\right)$ is comparable to the spin-down age, $\frac{P}{2 \dot{P}}$. In any evolutionary scenario, the white dwarf is formed after the primary. This suggests that in weak field pulsars such as PSR 0655+64 $\left(B, \sim 10^{10} \mathrm{G}\right)$ the magnetic field does not decay as rapidly as in the isolated, strong field pulsars (see Kulkarni (1986) for more details).

b)0820+02: A faint $\left(m_{R} \sim 22.8^{m}\right)$, blue $\left(\mathrm{V}-\mathrm{R} \sim-0.2^{m}\right)$ star is coincident with the position given by Manchester et al. 1983. A preliminary U-B determination by Liebert and Spinrad (pers. comm.) suggests the candidate is a hot white dwarf. Unlike the secondary. in $0655+64$, the white dwarf cooling age is much smaller than $\frac{P}{2 \dot{P}} \sim 10^{8} \mathrm{y}$ (see Kulkarni (1986) for further details). Consistent with this young age the field strength of the pulsar is $\sim 3.3 \times 10^{11} \mathrm{G}-$ not too different from that of a typical single pulsar.

c) $2303+46:$ The large eccentricity of this system immediately suggests that the secondary is another neutron star (Stokes et al. 1985) - consistent with the lack of optical detection $\left(m_{R} \gtrsim 23^{m}\right)$. These limits will be improved during the coming Fall season.

d)1855+09: In collaboration with S. Djorgovski we tentatively report a faint $\left(m_{V} \sim 23^{m}\right)$ star which is within $3 \sigma$ of the best timing position (Segelstein et al. 1986). Pending further optical data all we can say at this point is that the candidate (if not a chance background source) is consistent with the low-mass, old white dwarf.

\section{References}

Damashek et al. (1982), Ap. J. 253, L57.

Kulkarni, S. R. (1986), Ap. J. July issue.

Manchester, R. N. et al. (1983), Ap. J. 268, 832.

Segelstein, D. J. et al. (1986), Nature, in press. 